
temps de la convergence?

\title{
Annette Béguin-Verbrugge, Susan Kovacs (dir.) : Le cahier et l'écran. Culture informationnelle et premiers apprentissages documentaires
}

\section{Yolande Maury}

\section{Q OpenEdition}

\section{Journals}

Édition électronique

URL : http://journals.openedition.org/edc/3444

DOI : 10.4000/edc.3444

ISSN : 2101-0366

\section{Éditeur}

Université de Lille

Édition imprimée

Date de publication : 30 juin 2012

Pagination : 213-217

ISBN : 978-2-917562-07-9

ISSN : $1270-6841$

\section{Référence électronique}

Yolande Maury, « Annette Béguin-Verbrugge, Susan Kovacs (dir.) : Le cahier et l'écran. Culture

informationnelle et premiers apprentissages documentaires ", Études de communication [En ligne], 38 |

2012, mis en ligne le 01 janvier 2012, consulté le 22 septembre 2020. URL : http://

journals.openedition.org/edc/3444; DOI : https://doi.org/10.4000/edc.3444

Ce document a été généré automatiquement le 22 septembre 2020.

() Tous droits réservés 


\title{
Annette Béguin-Verbrugge, Susan Kovacs (dir.) : Le cahier et l'écran. Culture informationnelle et premiers apprentissages documentaires
}

\author{
Yolande Maury
}

\section{RÉFÉRENCE}

Annette Béguin-Verbrugge, Susan Kovacs (dir.) : Le cahier et l'écran. Culture informationnelle et premiers apprentissages documentaires, Hermès-Lavoisier (coll. Systèmes d'information et organisations documentaires ; Stéphane Chaudiron, dir.), 2011

1 L'ouvrage coordonné par Annette Béguin-Verbrugge et Susan Kovacs présente les résultats de la recherche menée en 2006-2010, par une équipe de six chercheurs de l'université Lille 3, dans le cadre de l'Équipe de Recherche Technologique en éducation (ERTé) «Culture informationnelle et curriculum documentaire ». L'objectif de l'équipe était, à partir de l'observation des pratiques info-documentaires dans un groupe scolaire de l'agglomération lilloise, d'étudier comment se construit un "regard documentaire " chez des enfants de la maternelle au CM2, et d'esquisser les fondements d'une culture informationnelle. Organisé en neuf chapitres, l'ouvrage propose une réflexion collective qui emprunte, suivant la sensibilité des chercheurs, aux sciences de l'information et de la communication mais aussi à la sociologie ou aux sciences de l'éducation.

2 La dynamique d'ensemble de l'ouvrage, comme le souligne le chapitre introductif, (pp.19-41) est de partir de l'analyse des contextes et des dispositifs pour aller à la rencontre des différents acteurs (élèves, enseignants, familles), étudiés à travers leurs usages et leurs pratiques, à la fois scolaires et personnels. Il s'agit également d'étudier, 
ainsi que le suggère le titre, les places respectives de deux objets à la symbolique forte, "Le cahier et l'écran ", très complémentaires dans le quotidien de l'école. Les auteurs ont fait le choix d'une approche anthropologique, en appui sur des observations ethnographiques, en immersion dans l'établissement lillois (observations in situ, dispositif automatisé de vidéo-traçage, entretiens). D’entrée, des précautions méthodologiques sont prises, et la question est posée de l'adéquation de la méthodologie à la finalité de l'étude: à savoir la possibilité d'élaborer un modèle général à partir de l'exploration d'un cas particulier, "considéré comme un 'microcosme' dont les traits s'appliqueraient forcément aux situations similaires » (p. 29).

3 Dans le chapitre 1 (pp. 43-62), Annette Béguin et Marie Desprès-Lonnet présentent le contexte matériel et humain de l'étude: le groupe scolaire et son environnement, l'agencement des espaces, les réseaux d'acteurs et de ressources, la gestion et la circulation des documents... Sont soulignées, en l'absence de personnel qualifié, les limites d'une gestion collective et empirique des ressources, et une sous-utilisation de ces ressources, notamment numériques, par manque de formation et de moyens.

4 Les quatre chapitres suivants, en appui sur les discours des enseignants, mettent en évidence une prééminence de la culture livre et une forte valorisation du support papier, tout en pointant un oubli de la matérialité documentaire lorsque la préoccupation première est l'accès à l'écrit.

5 Laurence Le Douarin, sociologue, analyse sous l'angle de l'évolution du rapport à la lecture au long du cursus (chapitre 2, pp.63-93), le rôle de la Bibliothèque Centre Documentaire (BCD) dans le processus d'acculturation aux documents. Attentive au rapport autonomie-contrainte, elle montre comment, du registre de l'émotion à celui de la compréhension du texte, se met en place un processus de standardisation qui favorise l'accès à une culture légitime mais peut venir en contradiction avec le besoin de singularisation et/ou d'autonomie des élèves.

6 Susan Kovacs, dans le chapitre 3 (pp.95-112), se place du côté de l'enseignant pour analyser les transformations de documents en jeu dans la création de supports pédagogiques. Porteuses d'une idéologie, ces transformations semblent davantage préoccupées des contenus pédagogiques et des objectifs à atteindre que du «sens des formes » documentaires. Il s'ensuit une valorisation du texte par rapport au document qui ne favorise pas l'acquisition d'une culture documentaire.

7 Annette Béguin-Verbrugge prolonge la réflexion avec l'étude des «vocabulaires documentaires » qui circulent dans l'école (chapitre 4, pp. 113-128) : peu nombreux, essentiellement référés à l'univers du livre et du texte écrit, et souvent étudiés en fonction de la nécessité pratique. Cette approche permet la mise en place de réflexes chez les élèves, mais la formation des concepts implique, selon l'auteur, que les enseignants soient davantage attentifs "à l'évolution des définitions proposées aux enfants, tant au plan linguistique qu'au plan encyclopédique ».

8 L'image occupe également une place très réduite dans la culture scolaire, note Laure Bolka-Tabary (chapitre 5, pp.129-151), qu'il s'agisse de réception ou de production, alors que son rôle pourrait être déterminant dans les apprentissages documentaires (aide à la recherche, à la prise d'information, catégorisation documentaire...). Ici aussi, ce sont le plus souvent les supports papier qui sont mis à contribution; l'audiovisuel, connoté loisirs, et le numérique sont peu exploités. Une sous-utilisation expliquée par 
des raisons culturelles (priorité à l'écrit) bien plus que par les contraintes matérielles ou pédagogiques qui ont cependant un rôle de renforcement.

Les trois chapitres suivants opèrent un déplacement du cahier à l'écran, et interrogent plus spécifiquement la place et le rôle du numérique, relativement au livre, à l'école et dans les familles.

10 C'est un état des lieux en demi-teinte que dresse le chapitre 6 (pp. 153-196), en appui sur le dispositif d'observation mis en place par Louis-François Claro: équipement ancien, maîtrise variable de l'outil par les enseignantes, difficultés de communication pédagogique face au numérique. Alors que les élèves sont censés obtenir la certification B2i École en fin de primaire, le potentiel du numérique est sous-exploité : le travail informatique apparaît coupé de la vie de classe, et centré sur les techniques et les procédures au détriment du sens des activités.

11 Pour ce qui est de la recherche d'information, étudiée par Marie Desprès-Lonnet dans le chapitre 7 (pp.197-230), les enseignantes l'abordent comme une familiarisation avec l'écrit, sans avoir forcément conscience des compétences documentaires en jeu et donc sans que ces compétences ne soient clairement explicitées. Une logique d'usage prévaut, à la recherche de repères opérationnels. Les documents sont peu questionnés, qu'ils soient numériques ou papier.

12 Par contraste, la place du numérique et du livre dans les familles (des familles plutôt favorisées), étudiée par Laurence Le Douarin (chapitre 8, pp. 231-248), donne à voir un équipement somme toute assez riche et des usages très divers. Mais si ces usages confirment la place importante accordée à la lecture chez les tout-petits, ils témoignent aussi de tensions et de variations dans les stratégies éducatives des parents (modes d'accès aux équipements, recours au numérique, attentes à l'égard de l'école).

13 L'ouvrage se termine avec le point de vue des élèves, analysé par Annette BéguinVerbrugge (chapitre 9, pp. 249-300), mettant en évidence la construction de compétences documentaires au-delà des seuls enseignements, via la fréquentation de la BCD ou les interactions sociales en famille et à l'école. L'école garde toutefois un rôle primordial quand il s'agit de structurer ces connaissances, notamment dans le domaine du numérique.

14 Avec ce compte rendu de recherche, l'équipe lilloise apporte un éclairage intéressant et attendu - sur les premiers apprentissages documentaires à l'école, un domaine jusqu'ici peu investi en France par la recherche en SIC. Le choix d'une observation ethnographique est particulièrement bienvenu, la parole des acteurs est restituée de manière précise, donnant vie aux pratiques documentaires. L'insistance sur certains thèmes au fil des chapitres (matérialité documentaire, priorité à l'écrit...) peut parfois donner une impression de redondance, mais le propos est clair, les auteurs ont retenu une approche didactique, chaque chapitre se terminant par une conclusion synthétique qui recentre sur les apports à la problématique principale. Une bibliographie de 14 pages complète l'ensemble.

15 Sur le fond, sans proposer un modèle général - puisque l'étude est limitée à un cas unique-, l'ouvrage donne des pistes pour la mise en œuvre d'une pédagogie documentaire à l'école: une pédagogie ouverte, faisant une place au doute et au questionnement, et privilégiant une approche communicationnelle des savoirs. Les concepts-clés présentés, à partir desquels construire un regard docu-mentaire, débordent de la recherche documentaire pour prendre en compte les médias et les 
formes documentaires; ils sont référés, pour beaucoup, à l'univers du livre et du document papier, la continuité entre " le cahier et l'écran » est moins prise en compte. Pour finir, nous remarquerons que l'attention portée à la matérialité documentaire dans l'acculturation à l'écrit, conduit à un focus sur la question de "littératie ", et la "culture documentaire» en tant que composante de la «culture informationnelle», telle que définie en introduction, dans un sens large.

L'ouvrage devrait intéresser les chercheurs, mais aussi les professionnels de l'information-documentation, les enseignants, et les responsables institutionnels intéressés par la problématique de la culture informationnelle.

\section{AUTEURS}

\section{YOLANDE MAURY}

MCF en Sciences de l'Information et de la Communication, Laboratoire GERiiCO (EA4073) -

Université de Lille 3 